\title{
Craquelures et art : le temps et la matière
}

Frédérique Giorgiutti-Dauphiné (giorgiutti@fast.u-psud.fr) et Ludovic Pauchard

Laboratoire FAST (Université Paris-Saclay et CNRS UMR 7608),

Bâtiment Pascal (bât. 530), rue André Rivière, Campus universitaire, 91405 Orsay

\section{On s'intéresse à la matière}

qui constitue une peinture d'art,

c'est-à-dire une succession

de couches picturales déposées

sur un support. Cet ensemble est

complexe de par sa composition

et sa géométrie (différentes

couches superposées). Il est

le siège de nombreux phénomènes

physiques et chimiques

qui apparaissent à plusieurs

moments dans la vie de l'œuvre.

Différents types de dégradations

altèrent ou modifient

la perception du tableau au cours

du temps. Les craquelures

sont parmi les plus apparentes.

Leur étude permet de caractériser

certaines propriétés mécaniques

de la matière constituant

la peinture et ainsi de révéler

de nombreux secrets sur l'œuvre.

Le réseau de craquelures

constitue donc « l'empreinte

digitale d'un tableau » et en cela

peut aider à authentifier

des œuvres.
Une peinture d'art est un système complexe de par sa géométrie, mais également sur le plan physico-chimique. En effet, un tableau est une succession de couches de peinture de différentes épaisseurs (couches picturales) déposées sur un support. Les supports les plus classiques restent la planche de bois qui a pris son essor au Moyen Âge, supplantée à la Renaissance par les toiles, plus légères, qui permettent ainsi de réaliser des peintures de dimensions plus importantes et moins sensibles aux variations hygrométriques. Une couche de préparation recouvre le support, afin de le rendre plus lisse et d'améliorer l'adhésion de la peinture, et une couche de vernis protège les couches picturales.

La composition de la peinture est ellemême complexe, les formulations sont très diverses et varient d'un produit à l'autre. Les composants principaux sont les pigments (oxydes ou sels métalliques), d'origine organique ou végétale, et le liant. Ce dernier est généralement une huile ou une émulsion aqueuse de polymères acryliques. Différents additifs tels que des tensioactifs, des solvants ou des plastifiants sont incorporés à cette base afin de modifier sa viscosité, ses propriétés mécaniques ou encore sa stabilité. Les huiles sont constituées de triglycérides qui vont subir un séchage chimique, c'est-à-dire qu'elles vont former un réseau tridimensionnel (un film solide) par des processus d'oxydation puis de polymérisation. Dans le cas des émulsions aqueuses, le séchage est physique et lié à l'évaporation de l'eau.

L'ensemble constituant la peinture subit de multiples contraintes mécaniques au cours du temps. Ces dernières peuvent provenir des variations environnementales (hygrométrie, température, oxydation, radiations UV...) ou des processus physiques et chimiques opérant lors du séchage et dans le temps (vieillissement). Le support peut également se déformer et ainsi générer des contraintes supplémentaires dans la couche picturale. Enfin, les conditions de conservation d'un tableau (chocs éventuels, enroulements...) et les diverses opérations de restauration peuvent aussi être sources de contraintes. Les conséquences de l'ensemble de ces contraintes sont multiples; elles sont responsables des dégradations observées sur un tableau comme, par exemple, des altérations optiques (changement de couleur ou de texture) ou des altérations mécaniques, telles que les craquelures.

La diversité des constituants des peintures ainsi que la complexité liée à leur structure multicouche conduisent à une grande variabilité dans les morphologies des figures de craquelures observées. Ainsi, les craquelures constituent le premier signe du vieillissement d'une œuvre, et forment "l'empreinte digitale " du tableau en révélant les propriétés mécaniques de la matière ou en donnant des informations sur les méthodes employées par l'artiste. En cela, elles peuvent aider à l'authentification d'une œuvre en apportant des informations complémentaires à celles obtenues par d'autres techniques. L'étude des morphologies de craquelures est une technique non invasive qui a permis de distinguer, dans une série de tableaux de Georges de la Tour, les originaux des copies [1] : les réseaux de craquelures dans les copies présentaient des morphologies très différentes de celles des originaux. 


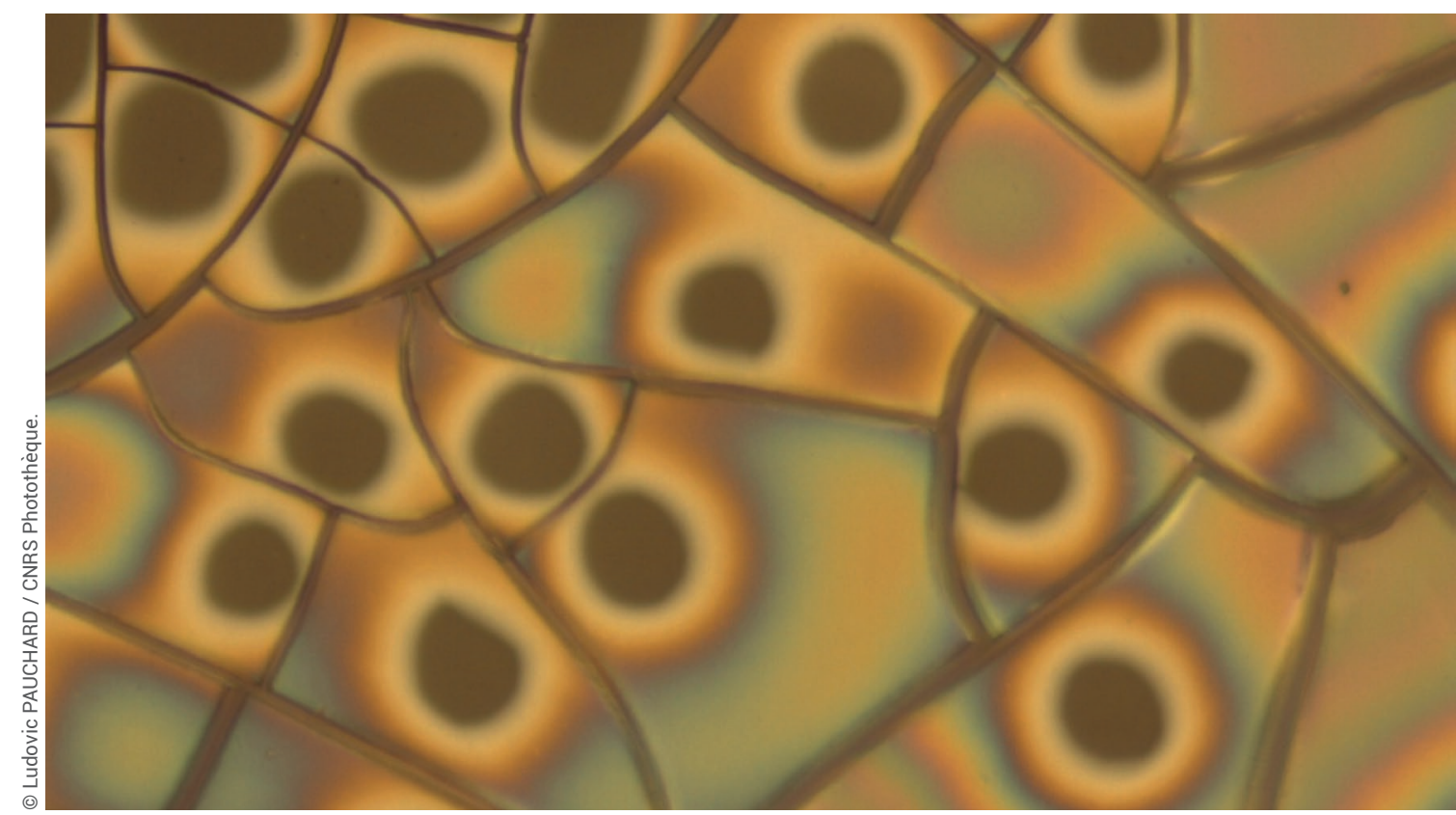

Figures de craquelures induites par séchage d'une couche de particules colloïdales. Les craquelures forment des polygones plus ou moins réguliers, qui se décollent ensuite partiellement. Les disques sombres à l'intérieur de chaque polygone correspondent aux régions d'adhésion entre la couche et le substrat. Ces disques sont entourés de franges d'interférence caractérisant la courbure des polygones décollés (largeur de l'image $100 \mu \mathrm{m}$ ). L'intérêt est de modéliser expérimentalement les morphologies de craquelures dans des systèmes modèles afin de reproduire les craquelures dans les couches picturales.

\section{Les systèmes modèles et la caractérisation mécanique}

Une craquelure se forme dans un matériau sous contraintes mécaniques, à partir d'un défaut. On peut noter qu'une modification de l'organisation des pigments au cours du vieillissement peut être à l'origine de défauts qui seront ainsi le point de départ de futures craquelures. La propagation d'une craquelure permet de relaxer les contraintes en son voisinage.

Une fois la craquelure initiée, sa propagation nécessite de l'énergie élastique. Cette énergie est proportionnelle au volume de matière contraint et donc à l'épaisseur de la couche fracturée. Ainsi, pour des épaisseurs de couches inférieures à une épaisseur critique $h_{c}$ (de l'ordre de quelques micromètres), aucune craquelure ne se forme du fait du déficit d'énergie élastique nécessaire à sa propagation. Outre l'épaisseur, il faut prendre en compte les propriétés mécaniques du matériau afin de déterminer son comportement sous contraintes.
Pour mieux comprendre les mécanismes de fracturation, nous les avons modélisés par des systèmes dont on peut contrôler les propriétés mécaniques. Ces systèmes sont des suspensions aqueuses de nanoparticules déposées en couche mince (une seule couche) sur un substrat indéformable et non poreux (une lame de verre). Un exemple est montré sur la photo ci-dessus.

Ces suspensions sont constituées d'un mélange de nanoparticules dures et molles (déformables). En modifiant la fraction volumique en particules molles, on peut obtenir un matériau qui, en séchant, deviendra plus ou moins fragile ou ductile. Le caractère fragile / ductile dépend du domaine dans lequel a lieu la fracturation du matériau. Ainsi, comme représenté sur la figure 1a, une rupture dans le domaine élastique (partie linéaire de la courbe contrainte-déformation où les déformations sont réversibles) est caractéristique d'un matériau fragile, tandis qu'une rupture dans le domaine plastique (domaine où les déformations sont irréversibles) est la signature d'un matériau ductile. Dans un matériau fra- gile, la propagation de la craquelure est généralement rapide et n'implique aucun mécanisme d'endommagement ; le matériau est indemne juste avant la rupture. Dans une peinture, cette dernière est généralement causée par une rupture en chaîne des molécules sans glissement entre elles. Les surfaces de la craquelure sont nettes. Au contraire, dans un matériau ductile la rupture est plus lente, les surfaces de la craquelure sont accidentées et ne se recollent pas après rupture.

Des mesures de micro-indentation permettent d'appuyer sur le matériau avec une pointe (ici sphérique) et de suivre la profondeur d'enfoncement de cette pointe avec le temps. Il est aussi possible d'appliquer une force croissante et de suivre la déformation induite dans la matière. Dans le domaine élastique, la courbe obtenue peut être ajustée grâce à un modèle théorique décrivant le contact entre une pointe et un matériau (modèle de Hertz). On peut alors en déduire le module élastique du matériau. Lorsque la courbe expérimentale s'écarte de cet ajustement théorique, le matériau devient plastique et il est alors possible d'identifier 

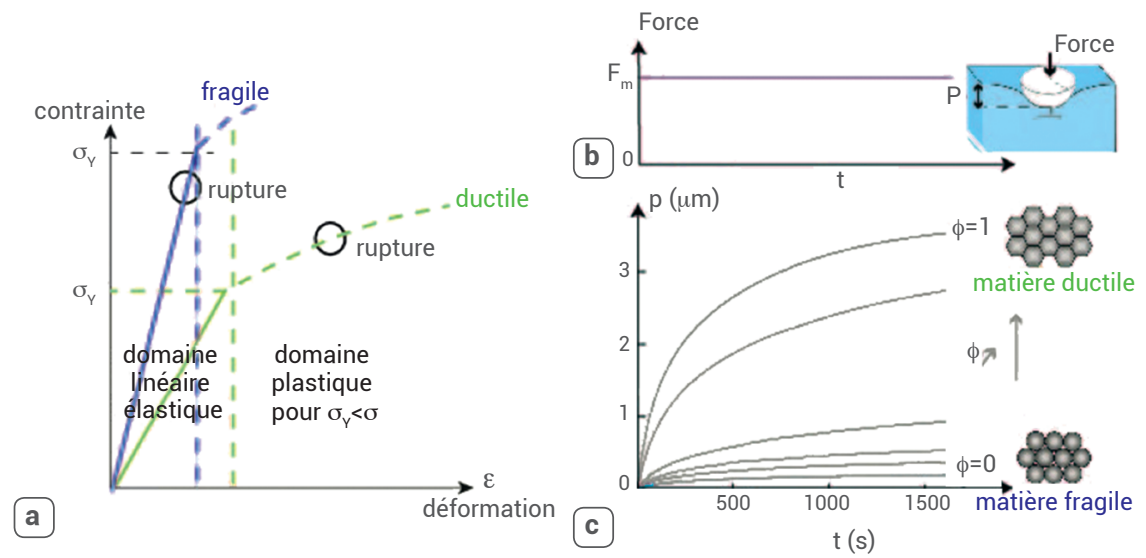

1. Caractérisation des propriétés mécaniques des matériaux.

(a) Comparaison des courbes d'évolution de la contrainte $\sigma$ en fonction de la déformation $\varepsilon$ pour un matériau fragile (courbe bleue) et un matériau ductile (courbe verte). La partie linéaire correspond au régime élastique. La limite entre les domaines élastique et plastique est donnée par la contrainte critique $\sigma_{\gamma}$.

(b) Caractérisation mécanique par technique de micro-indentation. Cette technique consiste à faire pénétrer une pointe, ici sphérique, dans la matière avec une force contrôlée notée $F_{m}$ et à mesurer la profondeur $\mathrm{p}$ de pénétration dans le matériau.

(c) Évolution temporelle de $p$ dans des couches modèles dont on contrôle les propriétés mécaniques en mélangeant des particules dures et molles; $\phi$ représente la fraction volumique des particules molles. Pour la matière ductile ( $\phi$ élevé), la réponse à l'application de la force prend un certain temps (composante visqueuse)

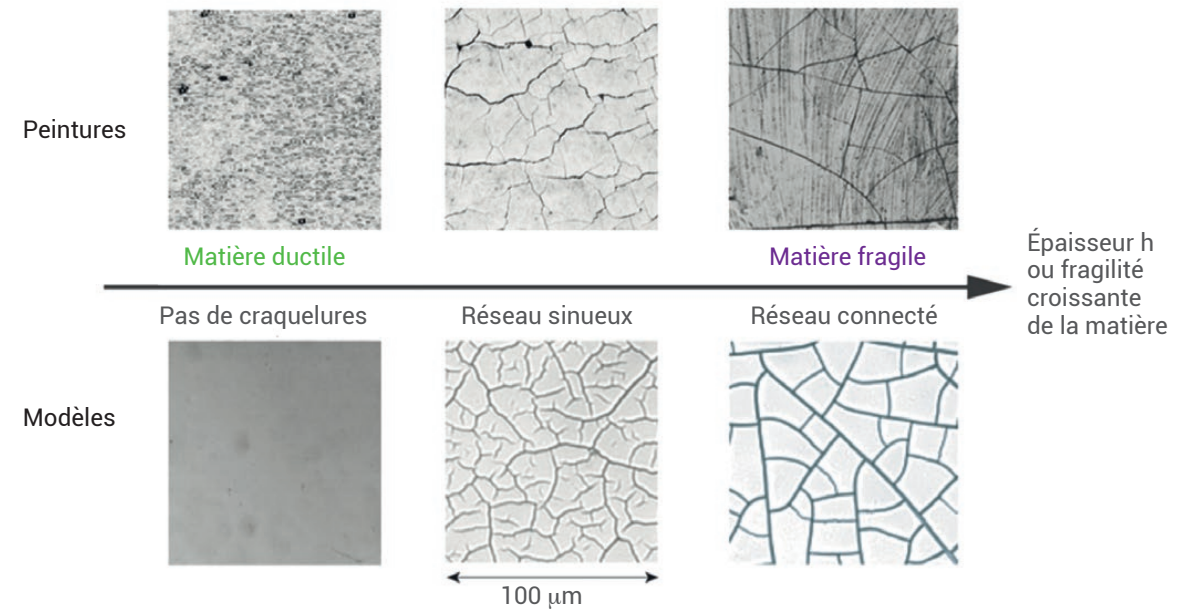

2. Les différents réseaux de craquelures en fonction des deux paramètres qui agissent de la même manière sur la formation des craquelures : l'épaisseur des couches et les propriétés mécaniques (fragilité, ductilité), dans les peintures (en haut) et dans les systèmes modèles obtenus par séchage (suspensions aqueuses de nanoparticules déposées sur une lame de verre assurant une bonne adhésion entre support et suspension, en bas).

$\gg>$

la contrainte critique. Un autre type d'expérience de micro-indentation consiste à imposer une force constante et à enregistrer l'évolution temporelle de la déformation (expérience de fluage, voir les figures $1 \mathrm{~b}$ et $1 \mathrm{c}$ ). On peut ainsi déterminer la dynamique de réponse du matériau à l'application de la force et estimer une composante visqueuse.

En modifiant les compositions de ces systèmes modèles, à l'instar de ce qui se produit dans une peinture, il est donc possible d'obtenir des matériaux plus ou
On observe que les réseaux dits " sinueux " se forment soit dans des matériaux ductiles, soit dans des couches de faible épaisseur présentant des défauts. Les craquelures ne sont pas rectilignes et sont courtes, d'où la dénomination "sinueux ». Les réseaux dits " connectés " sont pour leur part caractéristiques des matériaux fragiles ou peuvent se rencontrer dans des couches d'épaisseurs importantes. Les craquelures sont rectilignes, elles forment un réseau fermé. Ces réseaux sont de même nature que ceux observés sur des céramiques anciennes, par exemple (fig. 4). Dans tous les cas, la formation de craquelures nécessite la présence de défauts dans la matière.

Il est possible d'effectuer des prélèvements sur les peintures (sur les bords du tableau) afin d'évaluer par mesures d'indentation les propriétés mécaniques des différentes couches. Suivant les pigments utilisés, le module élastique peut varier de plus de trois ordres de grandeur et la rigidité d'une couche augmente généralement avec son âge, comme le suggèrent des expériences réalisées sur des peintures modèles sur une quinzaine d'années. Le vieillissement de la matière, l'environnement, le caractère multicouche ou encore la déformation du support sont autant de sources de modifications de la matière et de contraintes qui vont générer des réseaux dont les morphologies sont diverses et complexes.

Bucklow [3] a proposé une classification simplifiée, fondée sur l'observation des différentes écoles de peinture à travers les siècles et leur origine géographique (France, Flandre, Allemagne et Italie). Il a ainsi pu extraire un nombre réduit de critères caractérisant un réseau de craquelures, comme la forme de ces dernières, leur densité, ou le type de connections...

Visuellement, en isolant une craquelure, on peut différencier, indépendamment du type de réseau, deux grandes familles de craquelures: les craquelures prématurées, aussi dites "de séchage ", et les craquelures d'âge. Les premières se forment dès le début de la vie de la peinture, généralement au cours de la consolidation de la couche picturale, par un séchage chimique ou physique. On a alors fissuration d'un matériau ductile. Ces craquelures prématurées sont dues à un défaut de mise en œuvre de la peinture, à une consolidation trop rapide liée à la matière utilisée ou à 

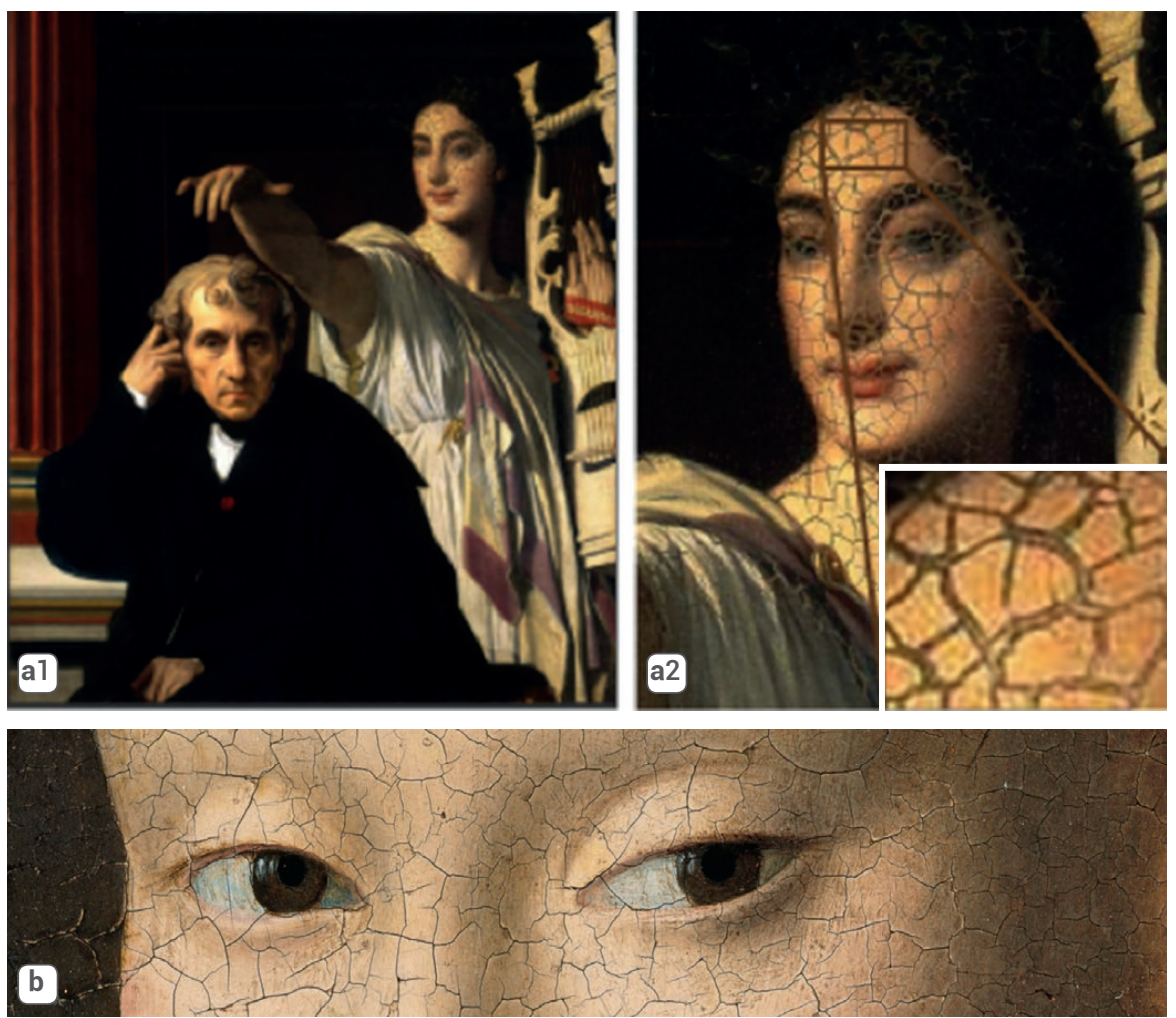

3. Craquelures prématurées et craquelures d'âge.

(a1) Luigi Cherubini et la Muse de la poésie lyrique, Ingres (1842), huile sur toile, Musée du Louvre (Wikimedia Commons).

(a2) Le visage de la Muse révèle ses craquelures prématurées, très ouvertes, présentes dans le front (agrandies dans l'insert, en bas à droite).

(b) Portrait d'une jeune fille, Petrus Christus (vers 1470), huile sur bois de chêne, Gemäldegalerie, Berlin : on observe de fines craquelures d'âge se formant au cours du vieillissement dans un matériau fragile. (Wikimedia Commons. Photo : www.google.com/culturalinstitute/asset-viewer/UAGsuoFcmmRiTg / Google Art Project).

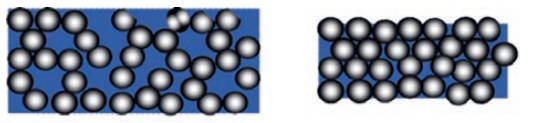

Rétraction de la couche induite par évaporation de solvant
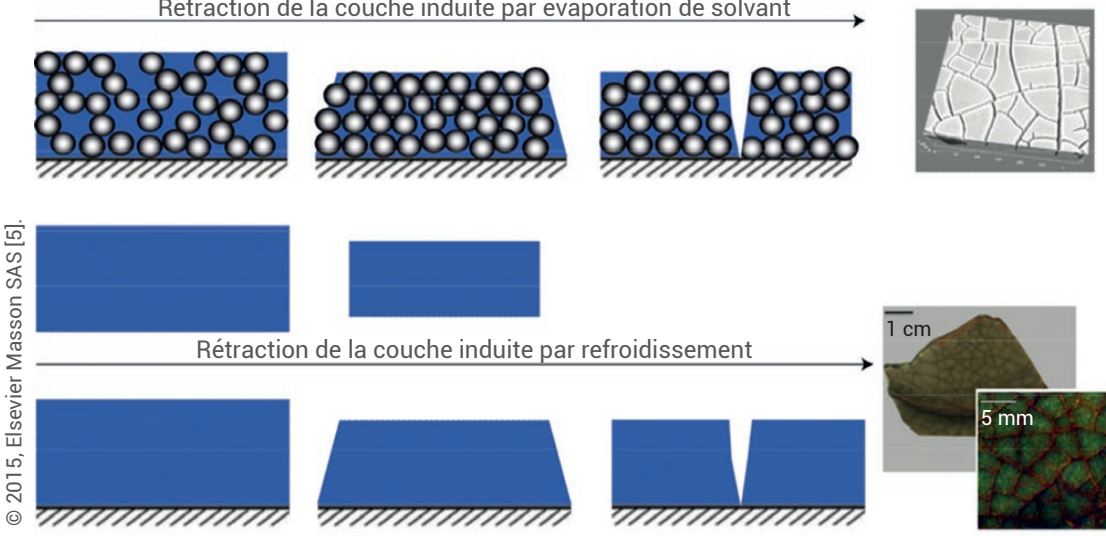

$\mathrm{T}_{0}$
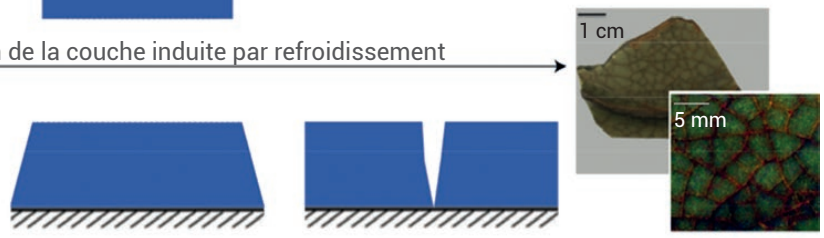

$\mathrm{T}_{1}<\mathrm{T}_{0}$

4. Schémas représentant les mécanismes conduisant à la formation d'une craquelure.

(a) Dans une couche libre (en haut), constituée de pigments et d'interstices remplis d'un solvant volatil qui sèche, la matière se rétracte. Dans le cas avec substrat (en bas), la rétraction à l'interface de la couche et de l'air est contrariée par la forte adhésion de la couche avec le support : il en résulte une déformation différentielle qui est relaxée par la formation de craquelures de séchage.

(b) Couche d'un matériau soumis à une diminution de température, dans une situation sans substrat (rétraction) et sur substrat (formation de craquelures). Photos : élément d'une céramique chinoise des dynasties Song et Yuan (960-1368, Shanghai Institute of Ceramics) et image agrandie obtenue par traitement d'image du réseau de craquelures. l'impatience du peintre qui n'a pas respecté, par exemple, la règle du « gras sur maigre " (qui postule qu'il faut attendre suffisamment de temps avant de déposer une nouvelle couche afin de s'assurer que la couche inférieure est totalement sèche). Une peinture d'Ingres montre un tel exemple (fig. 3a1). On sait que l'artiste a laissé le soin de terminer le visage de la Muse à son disciple de l'époque. Ce dernier n'a pas utilisé les mêmes compositions de peinture, en prenant un liant sans doute trop chargé en huile et en bitume (très fréquemment utilisé à l'époque). La consolidation a conduit à la formation de craquelures très ouvertes (fig. 3a2), caractéristiques de craquelures de séchage. La pénétration de ces craquelures est en général limitée à une ou deux couches au maximum. Leur grande ouverture résulte de la rétraction importante de la couche, qui va glisser sur la couche inférieure [2].

Ces craquelures prématurées peuvent aussi influencer le tracé de celles qui apparaitront plus tard, les craquelures d'âge. Ces dernières se forment au cours $\mathrm{du}$ vieillissement de la peinture. Elles sont fines et se développent dans l'ensemble de la stratigraphie de la couche picturale (fig. 3b). Elles sont associées à une matière fragile et rigide (de module élastique élevé), qui casse dans le domaine élastique. En vieillissant, la matière devient généralement de plus en plus fragile. Visuellement et sans faire aucun prélèvement, il est ainsi possible de discriminer les craquelures : si elles présentent des ouvertures importantes, il s'agira de craquelures prématurées, et si elles sont très fines, de craquelures d'âge.

\section{La formation des craquelures}

Les systèmes modèles (suspensions de nanoparticules) permettent de comprendre les différents mécanismes physiques à l'œuvre lors du séchage et en particulier la formation d'un réseau de craquelures. Lors du séchage d'une peinture, le solvant s'évapore, la concentration en particules solides augmente. Des contraintes se développent dans le matériau, générées par de fortes pressions capillaires liées aux ménisques air / liquide qui apparaissent suite à la disparition du solvant. Les contraintes ne sont alors pas constantes dans l'épaisseur, mais plus fortes au voisinage de l'interface avec le support. Sous 


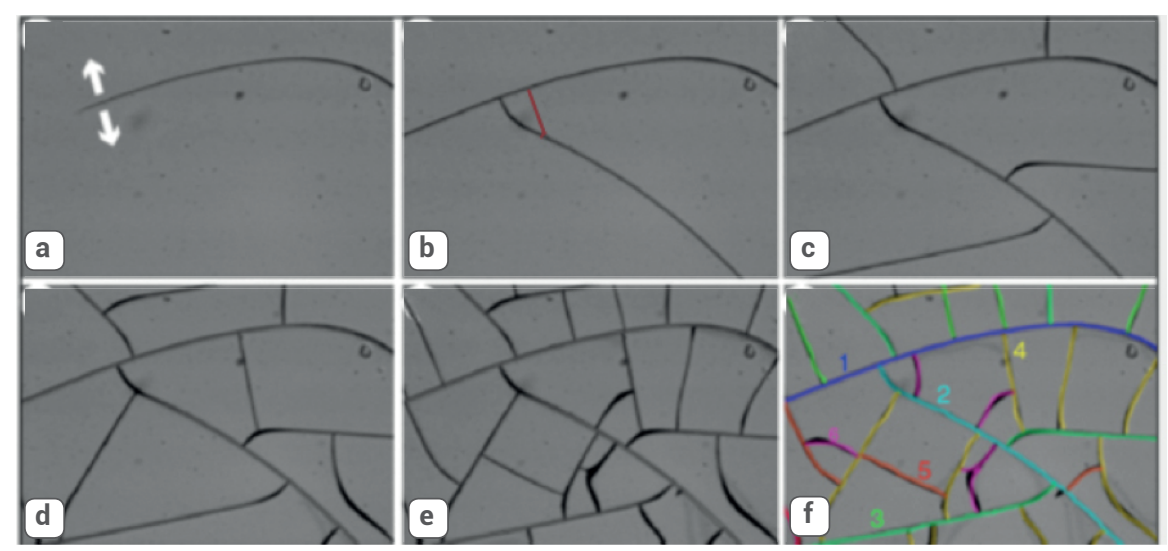

5. Formation hiérarchique d'un réseau de craquelures dans une couche picturale modèle [6] La trajectoire de chaque craquelure est guidée par un champ de tensions résidant et évoluant dans la couche picturale. Les flèches blanches illustrent ces tensions dans l'image (a).

(f) Figure finale présentant différentes générations. Chaque couleur correspond à une génération de craquelures : bleu pour la première génération, turquoise pour la seconde, vert pour la troisième, jaune pour la quatrième, rouge pour la cinquième et violet pour la dernière. (Largeur d'un cliché : $1 \mathrm{~mm}$ ).

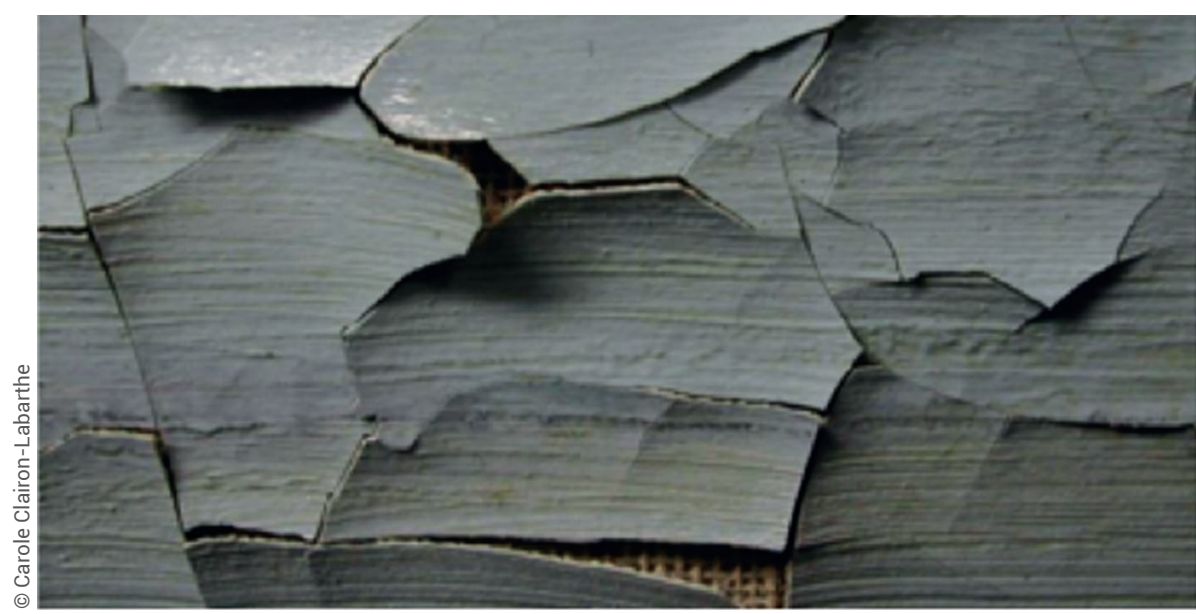

6. Décollement partiel d'une couche picturale du support.

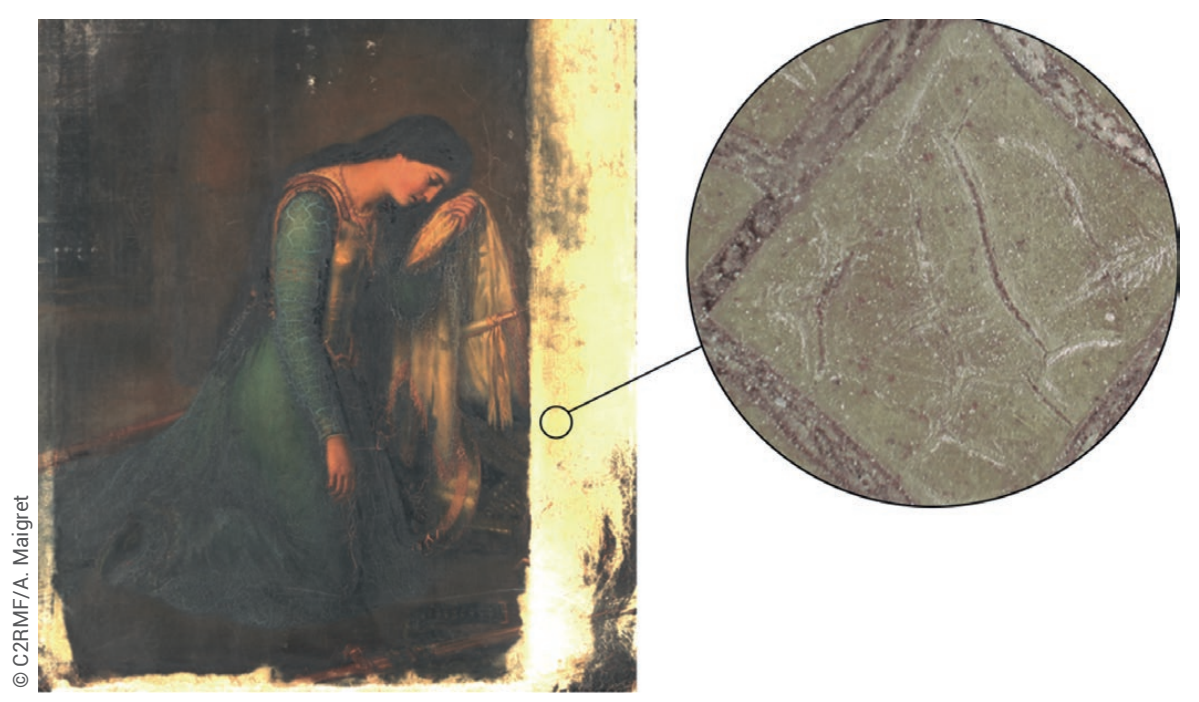

7. Jeanne d'Arc en prison, Louis Crignier, 1824, huile sur toile (Musée de Picardie, Amiens) En insert, grossissement dans la partie blanchie, montrant la présence de microcraquelures (diamètre de la zone grossie : $1 \mathrm{~mm}$ ).
$>>$

l'effet de ces contraintes, la matière se rétracte (haut de la fig. 4a). Cependant, si elle adhère au support ou à une souscouche, la rétraction est frustrée par l'adhésion qui empêche la couche de se déformer librement. Ceci conduit au développement d'une contrainte différentielle ; lorsque celle-ci atteint la contrainte critique de fracturation, le système relaxe les contraintes en créant des craquelures (bas de la fig. 4a). On notera que ce scénario est responsable de la formation des craquelures prématurées liées au séchage.

Le scénario est le même dans le cas d'une variation de température (fig. 4b), et ce processus est responsable des craquelures observées dans les céramiques. En particulier, les vases chinois de la dynastie Song ont une valeur particulière du fait des réseaux de fines craquelures obtenues lors du refroidissement (photos à droite de la figure 4b) [5], qui ont pour origine les forts gradients thermiques.

La propagation d'une craquelure dans une couche est guidée par les tensions du milieu (fig. 5a). Une fois propagée, sa trajectoire reste inchangée, mais le champ de contraintes au voisinage de la craquelure est modifié. Quand une autre craquelure s'approche de la première, sa propagation est modifiée de manière à prendre en compte les changements de contraintes dus à la première craquelure, elle se connecte alors perpendiculairement à celle-ci (fig. 5b). Le processus se poursuit ainsi. Les craquelures n'interviennent pas simultanément mais envahissent le plan de la couche par générations successives, délimitant ainsi des fragments plus ou moins réguliers (figures $5 c, 5 d$ et $5 \mathrm{e}$ ). À la fin du processus, six générations de craquelures sont observables sur la figure $5 \mathrm{f}$. On peut retracer approximativement l'histoire de la formation de ce réseau de craquelures : les craquelures les plus anciennes sont les plus longues; les deuxièmes générations se connectent aux premières... Les craquelures se forment ainsi de manière hiérarchique et constituent des chemins plus ou moins sinueux, avec une structure ordonnée ou non [6].

On peut ainsi répertorier les principaux paramètres caractérisant quantitativement un réseau de craquelures dans une couche picturale :

- la taille des fragments qui croît avec l'épaisseur de la couche picturale ; 
- l'ouverture ou la largeur d'une craquelure liée à l'aptitude ou à la résistance à la rétraction de la matière [2] ;

- la distance à partir de laquelle la trajectoire d'une craquelure est modifiée lors d'une connexion avec une craquelure préexistante (trait rouge noté d sur la figure $5 b)$.

Ces paramètres ont permis d'obtenir des hypothèses sur les propriétés mécaniques et les épaisseurs des couches de La Joconde et de La Belle Ferronnière [7].

D'autres craquelures, plus marginales, révèlent également des éléments d'histoire de la peinture : des chocs, conduisant à des craquelures concentriques, des craquelures parallèles entre elles le long de l'axe d'enroulement de certaines peintures sur toile qui étaient ainsi transportées [1], etc.

\section{Conservation et restauration}

Les opérations de restauration sont essentielles et nécessaires afin de stopper, d'estomper ou de réparer les dommages du temps.

En ce qui concerne les altérations mécaniques, il existe d'autres types de craquelures que celles discutées précédemment, par exemple les craquelures interfaciales entre deux couches qui induisent une perte d'adhésion. Ainsi, des fragments de peintures se décollent, s'incurvent pour former des réseaux de craquelures en cuvette (fig. 6) où des morceaux entiers de couche picturale peuvent de ce fait disparaitre et tomber. Pour retrouver de l'adhésion, les restaurateurs utilisent souvent des adhésifs dissous dans des solvants.

Plus globalement, l'utilisation de solvants en restauration est largement répandue, notamment lorsqu'il faut dissoudre un vernis jauni ou retirer les salissures sur une œuvre. Il en résulte une pénétration résiduelle du solvant dans la couche picturale, qui impactera cette dernière à plus ou moins long terme. Il est donc important de comprendre l'impact de certaines méthodes de restauration sur les modifications mécaniques des peintures.

Un autre type de dommage important est celui observé dans des cas de fortes hygrométries, ou lors d'un dégât des eaux lorsque de l'eau ruisselle sur un tableau. On observe alors des zones fortement blanchies : les « chancis » (fig. 7). Ce blanchiment est dû à des microporosités formées lors de la pénétration
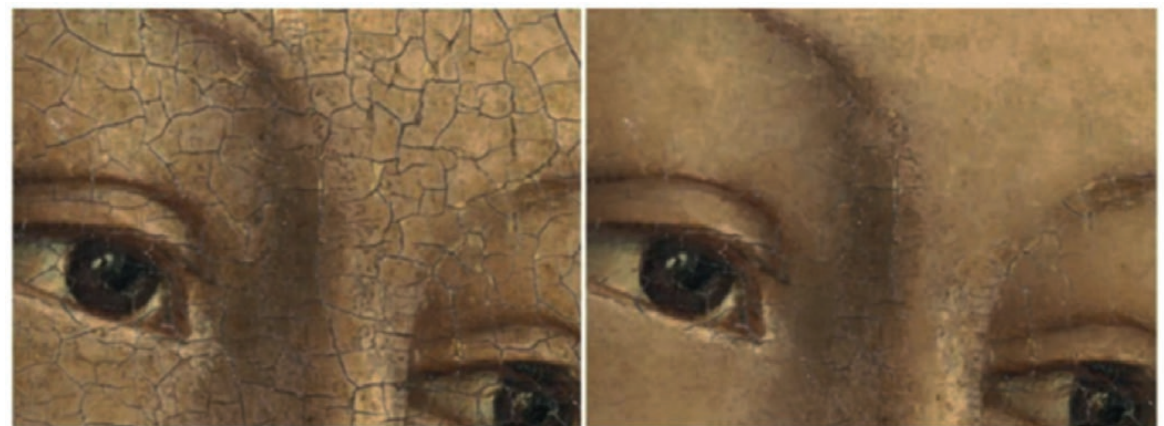

8. Détail de l'archange Gabriel, tiré de L'adoration de l'agneau mystique, Cathédrale Saint-Bavon de Gand (๑ www.lukasweb.be - Art in Flanders vzw, photo KIK-IRPA).

À droite : résultat d'un lissage extrait de [9].

de l'eau dans la couche picturale. L'opacité résulte de la diffusion de la lumière par ces microporosités. Ainsi la présence de craquelures, qui constituent un réservoir pour l'eau, affectera fortement la formation de chancis.

\section{Conclusion}

On comprend que les procédés de restauration ont un impact sur les propriétés mécaniques des matériaux. Celles-ci peuvent potentiellement jouer sur la stabilité du réseau de craquelures. Ainsi, un réseau de craquelures n'est pas une structure figée dans le temps. Il évolue avec les contraintes extérieures et peut aussi influer sur l'apparition et le développement d'autres types de dégradations comme les chancis [8]. De manière générale, toute action de restauration sur une œuvre va modifier la perception que nous en avons. En particulier, cette perception est fortement corrélée aux réseaux de craquelures, comme on peut le constater sur la figure 8. Les craquelures perturbent notre appréciation de l'image ; elles sont assimilées à un réseau de lignes de différentes épaisseurs et longueurs, plus ou moins contrastées, qui se superposent à l'image, affectant ainsi l'effet de profondeur de celle-ci. Elles traduisent également l'histoire de l'œuvre et lui conferent une valeur d'ancienneté et d'authenticité. Lors d'une restauration, un important débat consiste à déterminer si le réseau de craquelures doit être laissé tel quel, être estompé ou totalement supprimé.

Si une peinture est une œuvre d'art qui nous inspire et nous émeut, elle peut également être le lieu où se rencontrent la physique, la chimie, les sciences du patrimoine et l'histoire de l'art.

\section{Références}

1. L. Pauchard et al., « Craquelures dans les couches picturales de peintures d'art ", Reflets de la Physique 3 (2007) 5-9.

2• M. Leang et al., "Crack opening: from colloidal systems to painting", Soft Matter, 13 (2017) 5802-5808.

3. S. Bucklow, "The description of craquelure patterns", Studies in Conservation 42 (1997) 129-140.

4• F. Giorgiutti-Dauphiné et L. Pauchard, "Painting cracks: a way to investigate the pictorial matter", Journal of Applied Physics 120 (2016) 065107.

5. S. Lahlil et al., "Influence of manufacturing parameters on the crackling process of ancient Chinese glazed ceramics", Journal of Culture Heritage, 16 (2015) 401-412.

6. S. Bohn et al., "Hierarchical crack pattern as formed by successive domain divisions: temporal and geometrical hierarchy",

Phys. Rev. E 71 (2005) 046214.

7· La Joconde, essai scientifique, ouvrage collectif sous la direction de C. Lahanier, Codex Images (2007).

8• A. Genty-Vincent et al., "Four flux model of the light scattering in porous varnish and paint layers: towards understanding the visual appearance of altered blanched easel oil paintings", Appl. Phys. A, 123 (2017), article 473.

9. L. Platisa et al., "Spatiogram features to characterize pearls and beads and other small ball-shaped objects in paintings", dans Vision and material: interaction between art and science in Jan Van Eyck's time (M. de Mes et al., ed.), pp. 315-329, KVAB Press, Brussel (2012). 